\title{
Infants born very preterm react to variations of the acoustic environment in their incubator from a minimum signal-to-noise ratio threshold of 5 to $10 \mathrm{dBA}$
}

\author{
Pierre Kuhn ${ }^{1,2}$, Claire Zores ${ }^{1,2}$, Thierry Pebayle ${ }^{1}$, Alain Hoeft' ${ }^{1}$, Claire Langlet ${ }^{2}$, Benoît Escande ${ }^{2}$, Dominique Astruc ${ }^{2}$ \\ and André Dufour ${ }^{1}$
}

INTRODUCTION: Very early preterm infants (VPIs) are exposed to unpredictable noise in neonatal intensive care units. Their ability to perceive moderate acoustic environmental changes has not been fully investigated.

RESULTS: Physiological values of the 598 isolated sound peaks (SPs) that were $5-10$ and 10-15 dB slow-response A (dBA) above background noise levels and that occured during infants'sleep varied significantly, indicating that VPIs detect them. Exposure to 10-15 dBA SPs during active sleep significantly increased mean heart rate and decreased mean respiratory rate and mean systemic and cerebral oxygen saturations relative to baseline.

DISCUSSION: VPIs are sensitive to changes in their nosocomial acoustic environment, with a minimal signal-to-noise ratio (SNR) threshold of 5-10 dBA. These acoustic changes can alter their well-being.

METHODS: In this observational study, we evaluated their differential auditory sensitivity to sound-pressure level (SPL) increments below 70-75 dBA equivalent continuous level in their incubators. Environmental (SPL and audio recording), physiological, cerebral, and behavioral data were prospectively collected over $10 \mathrm{~h}$ in $26 \mathrm{VPIs}$ (GA 28 (26-31) wk). SPs emerging from background noise levels were identified and newborns' arousal states at the time of SPs were determined. Changes in parameters were compared over 5 -s periods between baseline and the $40 \mathrm{~s}$ following the SPs depending on their SNR thresholds above background noise.

V ery early preterm infants (VPIs) are exposed to nosocomial environmental stimuli that differ from the stimuli they encounter in utero. This new "naturalistic" milieu, especially loud noise, may interfere with their neurodevelopment and growth (1-4). This has led to specific recommendations for permissible noise criteria levels in the neonatal intensive care unit (NICU) (5-7). These recommendations were based primarily on the evaluation of the effects of noise on the developing auditory system and well-being of newborns. Most studies were experimental. Preterm newborns were exposed to $5 \mathrm{~s}$ of high artificial sound $(8,9)$, including sound-pressure levels (SPLs) ranging from 80 to $100 \mathrm{~dB}(8)$ and to warbling tones of $100 \mathrm{~dB}$ (9). The most prevalent responses were an increase in heart rate (HR) $(8,9)$ proportional to the SPL of the stimulus and a tendency toward a decrease in respiratory rate (RR) (9). Few studies have evaluated the impact of noise in the NICU on newborns' physiological stability. Unfortunately, some of these studies measured the effect of acoustic environments quite different from those in the contemporary NICU (10) or did not include preterm infants (11) or VPIs (10). Moreover, some yielded questionable results because of study design, nonreporting of background noise, and the absence of a well-defined study population (e.g., inclusion of newborns with a wide range of gestational age, GA) (12). Despite these limitations, these studies showed that a high level of environmental noise, $>70-75 \mathrm{~dB}$ slow response A (dBA) equivalent continuous level (Leq), could disrupt infant stability.

Two recent studies have explored the effect of NICU noise on VPIs. One, which involved eight extremely-low-birth-weight infants, found no correlation between moderate NICU noise levels (50-60 dBA) and arterial blood pressure, although there were HR changes starting from 25 to $45 \mathrm{~s}$ after noise peaks (13). The second study found that 11 VPIs exhibited sympathetic arousal, as measured by skin conductance, in response to natural sound $>65 \mathrm{dBA}$ and with background noise $<55 \mathrm{dBA}$ (14). These studies, however, did not determine the differential auditory SPL sensitivity (signal-to-noise ratio, SNR) or the precise effects of acoustic changes (in response to SPL $<70 \mathrm{dBA}$ Leq) on the physiological well-being of these VPIs. Such findings would be essential to a better understanding of the ontogeny of auditory functions in VPIs, resulting in better adaptation of their acoustic environment to their sensory abilities and preferences and thereby yielding more precise noise recommendations for VPIs. We therefore conducted this prospective observational study using a "naturalistic" approach to answer to the following questions: (i) can VPIs hear nosocomial sound peaks

\footnotetext{
'Laboratoire d'Imagerie and Neurosciences Cognitives, Université de Strasbourg/Centre National de la Recherche Scientifique, Strasbourg, France; ${ }^{2}$ Service de Pédiatrie 2 , Pôle Médico-Chirurgical Pédiatrique, Hôpital de Hautepierre, Centre Hospitalier Universitaire de Strasbourg, Strasbourg, France. Correspondence: Pierre Kuhn (pierre.kuhn@chru-strasbourg.fr)
} 
(SPs) that are 5-10 dBA and/or 10-15 dBA above background noise?; (ii) how do VPIs physiologically react to them?; and (iii) do they alter the infants' well-being?

\section{RESULTS}

\section{Background Noise}

For all VPIs breathing room air, on nasal continuous positive airway pressure and mechanically ventilated, L90s were 49.6, 59.9, and 49.3 dBA Leq, respectively; L50s were 51.0, 62.9, and 51.53 dBA Leq, respectively; and L10s were $52.9,65.2$, and 54.8 dBA Leq, respectively.

\section{SPs}

A total of 1,957 SPs $\geq 5$ dBA above background noise were recorded, leading to a median [range] number of SPs per infant of 66 [10-249] and a mean (+SD) of $75( \pm 51)$. The mean hourly exposure to SPs was $7( \pm 5)$ per infant. Based on this quantification, we can estimate that VPIs are exposed to an average of 168 SPs per day outside their nursing time. A total of 1,442 SPs were isolated (i.e., no other occurrences during the following 40 s). Of these, 1,128 (78\%) SPs had SNR ranging between 5 and $10 \mathrm{dBA}, 241$ (17\%) had SNR ranging between 10 and 15 $\mathrm{dBA}$, and 73 (5\%) had SNR above $15 \mathrm{dBA}$. The median [range] baseline SPL before SPs for newborns breathing room air, on nasal continuous positive airway pressure, and mechanically ventilated were 50.1 [48.8-54.1], 59.3 [54.1-61.3] and 50.4 [48.4-53.7] dBA Leq, respectively. A spectral analysis of the main SPs showed that their fundamental frequencies $\left(\mathrm{F}_{0}\right)$ ranged from 100 (incubator's motor) to $2,730 \mathrm{~Hz}$ (syringe pump alarm). For some complex noise, the frequencies of the first $\left(F_{1}\right)$ or second $\left(F_{2}\right)$ harmonic were of greater amplitude than $\mathrm{F}_{0}$, with the highest ones (ventilator's humidifier alarm) reaching $4,336\left(\mathrm{~F}_{1}\right)$ and $6,504 \mathrm{~Hz}\left(\mathrm{~F}_{2}\right)$.

By determining the arousal states of the infants in the 10-s baseline preceding the occurrence of SPs, it was possible to classify the SPs again. A concordance between the two observers was initially obtained for $94.5 \%(1,294 / 1,369)$ of the SPs and for all of them after concomitant reevaluation. Of the 1,369 SPs in ranges 5-10 and 10-15 dBA, 213 (16\%) occurred in state $1,385(28 \%)$ in state $2,59(4 \%)$ in states $3-4,173(13 \%)$ in state 5 , and $463(34 \%)$ in state 6. Owing to technical problems, behavioral evaluations were not possible for the remaining 76 SPs (5\%). Because relatively few SPs had an SNR $>15$ dBA or were in states 3-4 and because newborns in states 5-6 were physiologically and/or behaviorally unstable and mainly produced the SPs themselves (i.e., crying or movement), we further analyzed only the data for the SPs with an SNR ranging from 5 to $15 \mathrm{dBA}$ and occurring during sleep in states 1 and $2(n=598)$.

\section{Reactivity to SPs in Active and Quiet Sleep States}

Physiological responses. During the entire time course of the response, we observed significant profiles of variations for only two parameters (see Figure 1 and Figure 2): a decrease in RR after SPs occurring in quiet sleep in range 5-10 dBA $\left(\mathrm{F}_{(9,162)}=3.10, P<0.002\right)$ and in $\mathrm{SaO}_{2}$ after SPs occurring in active sleep in range $10-15 \mathrm{dBA}\left(\mathrm{F}_{(9,135)}=3.94, P<0.001\right)$. No variation in any other parameter reached statistical significance. Nevertheless, when comparing baseline to minimum and maximum values in the $a^{\star}$ and $b^{\star}$ periods, significant variations in different parameters were observed after SPs with an SNR of 5-10 dBA (Table 1) and 10-15 dBA (Table 2), suggesting the infants' ability to detect these SPs. The means of the highest amplitude of significant variations per infant for minimum and maximum values as compared with baseline

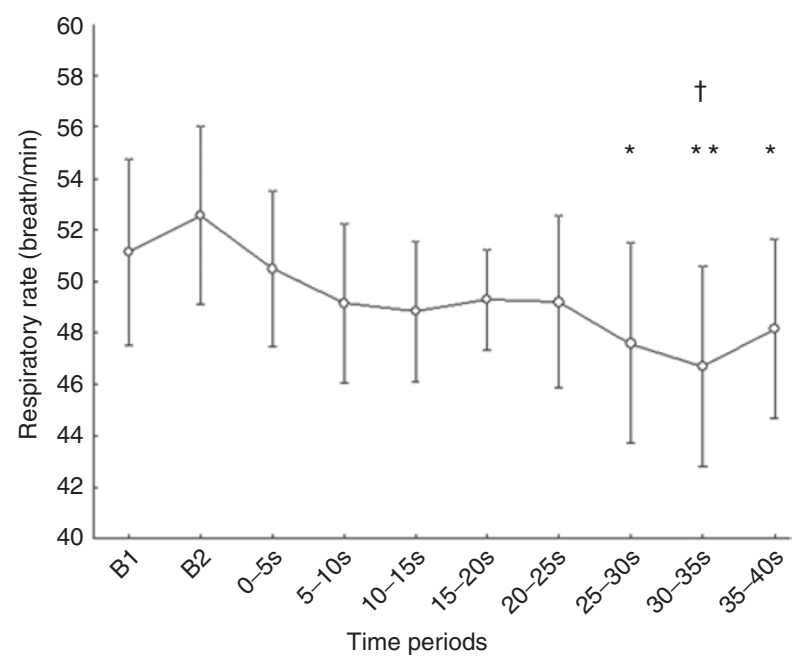

Figure 1. Variations of the respiratory rates following SPs occurring in quiet sleep, in the SNR range of 5-10 dBA Leq. Error bars represent $95 \%$ confidence intervals. B1 and B2 correspond to the baseline periods between -10 and $-5 \mathrm{~s}$ and between -5 and $0 \mathrm{~s}$ before SP occurrence, respectively. Post hoc analysis: ${ }^{*} P<0.05$ as compared with $B 2 .{ }^{*} P<0.01$ as compared with B2. ${ }^{+} P<0.05$ as compared with $\mathrm{B} 1$. SNR, signal-to-noise ratio; $S P$, sound peak.

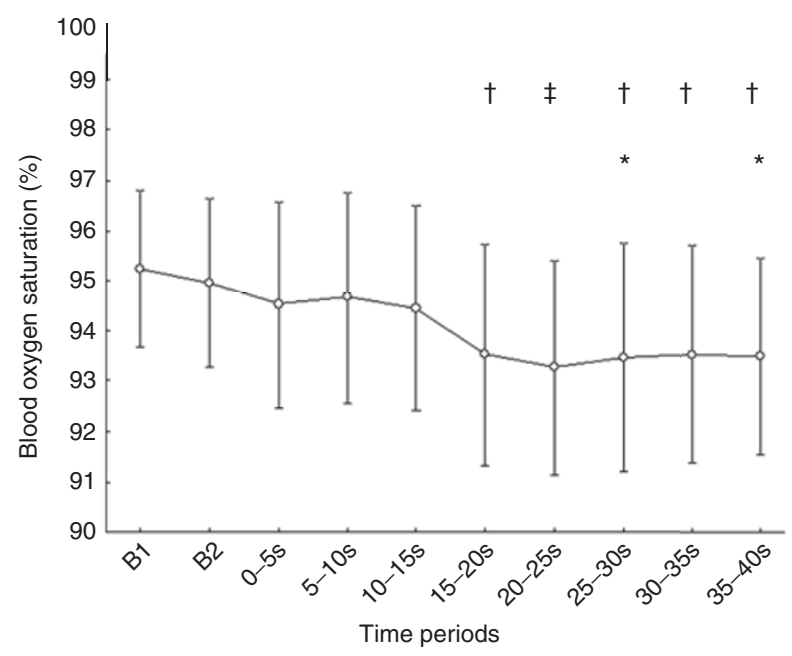

Figure 2. Variations of the blood oxygen saturations $\left(\mathrm{SaO}_{2}\right)$ following SPs occurring in active sleep in the SNR range of 10-15 dBA Leq. Error bars represent $95 \%$ confidence intervals. B1 and B2 correspond to the baseline periods between -10 to $-5 \mathrm{~s}$ and between -5 to $0 \mathrm{~s}$ before SP occurrence, respectively. Post hoc analysis: ${ }^{*} P<0.05$ as compared with $B 2 .{ }^{\dagger} P<0.05$ as compared with $B 1 .{ }^{\ddagger} P<0.01$ as compared with B1. SNR, signal-to-noise ratio; $S P$, sound peak. 
Table 1. Mean of the maximal variations relative to baseline and observed for each physiological parameter after SPs with an SNR of 5-10 dBA occurring in quiet $(n=182)$ and active $(n=336)$ sleep, depending on the time periods ( $a^{*}$ and $\left.b^{*}\right)$ after SPs

\begin{tabular}{|c|c|c|c|c|c|c|}
\hline \multirow[b]{2}{*}{ Parameter } & \multicolumn{3}{|c|}{ Quiet sleep (state 1) } & \multicolumn{3}{|c|}{ Active sleep (state 2) } \\
\hline & Variation (ANOVA) & $P$ value (post hoc) & $\begin{array}{l}\text { Average maximum } \\
\text { variation }( \pm S D)\end{array}$ & Variation (ANOVA) & $P$ value (post hoc) & $\begin{array}{l}\text { Average maximum } \\
\text { variation }( \pm S D)\end{array}$ \\
\hline \multirow[t]{2}{*}{ HR (beat/min) } & $\uparrow$ & $0.023\left(a^{*}\right)$ & $+2.4( \pm 3.4)$ & $\uparrow$ & $0.005\left(a^{*}\right)$ & $+2.3( \pm 3.9)$ \\
\hline & $P<0.001$ & $<0.001\left(b^{*}\right)$ & $+4.2( \pm 5.2)$ & $P=0.005$ & $0.011\left(b^{*}\right)$ & $+2.4( \pm 4.5)$ \\
\hline \multirow[t]{4}{*}{ RR (breath/min) } & $\downarrow$ & $<0.001\left(\mathrm{a}^{*}\right)$ & $-6.1( \pm 4.6)$ & $\downarrow$ & $0.005\left(a^{*}\right)$ & $-3.3( \pm 3.2)$ \\
\hline & $P<0.001$ & $<0.001\left(b^{*}\right)$ & $-7.3( \pm 4.5)$ & $P=0.003$ & $0.006\left(b^{*}\right)$ & $-2.9( \pm 6.0)$ \\
\hline & NS & NS $\left(a^{*}\right)$ & - & $\uparrow$ & $0.042\left(a^{*}\right)$ & $+1.9( \pm 3.4)$ \\
\hline & & $\mathrm{NS}\left(\mathrm{b}^{*}\right)$ & - & $P=0.029$ & $0.032\left(b^{*}\right)$ & $+2.4( \pm 5.0)$ \\
\hline \multirow[t]{2}{*}{$\mathrm{SaO}_{2}(\%)$} & NS & NS $\left(a^{*}\right)$ & - & $\downarrow$ & $0.039\left(a^{*}\right)$ & $-0.4( \pm 0.64)$ \\
\hline & & NS $\left(b^{*}\right)$ & - & $P=0.049$ & NS $\left(b^{*}\right)$ & - \\
\hline \multirow[t]{2}{*}{$\mathrm{rSO}_{2}(\%)$} & $\uparrow$ & $0.006\left(a^{*}\right)$ & $+0.64( \pm 0.87)$ & $\uparrow$ & $0.036\left(a^{*}\right)$ & $+0.5( \pm 0.7)$ \\
\hline & $P=0.004$ & $0.005\left(b^{*}\right)$ & $+0.57( \pm 0.79)$ & $P=0.046$ & $\mathrm{NS}\left(\mathrm{b}^{*}\right)$ & - \\
\hline \multirow[t]{4}{*}{ FTOE (\%) } & $\uparrow$ & NS $\left(a^{*}\right)$ & - & NS & NS $\left(a^{*}\right)$ & - \\
\hline & $P=0.014$ & $0.015\left(b^{*}\right)$ & $+0.8( \pm 0.2)$ & & NS $\left(b^{*}\right)$ & - \\
\hline & $\downarrow$ & $0.002\left(a^{*}\right)$ & $-0.7( \pm 0.8)$ & $\downarrow$ & $0.01\left(a^{*}\right)$ & $-0.6( \pm 0.8)$ \\
\hline & $P<0.001$ & $<0.001\left(b^{*}\right)$ & $-0.9( \pm 1.2)$ & $P=0.014$ & NS $\left(b^{*}\right)$ & - \\
\hline
\end{tabular}

FTOE, fractional cerebral tissue oxygen extraction; $\mathrm{HR}$, heart rate; $\mathrm{NS}$, not significant; $\mathrm{RR}$, respiratory rate; $\mathrm{rSO}_{2^{\prime}}$, regional cerebral oxygen saturation; $\mathrm{SaO}_{2^{\prime}}$ oxygen saturation; $\mathrm{SNR}_{\text {, }}$ signal-to-noise ratio; SP, sound peak.

$\downarrow, \uparrow$, Arrows indicate the direction of the observed variations, associated $P$ values indicate statistical significance (ANOVA for repeated-measure tests).

$a^{*}$ and $b^{*}$ indicate the periods ( $a^{*}: 0-20 \mathrm{~s}, b^{*}: 20-40$ s, post SP) in which a significant variation of the parameter was found in post hoc analysis (Newman-Keuls test) as compared with baseline.

Table 2. Mean of the maximal variations relative to baseline and observed for each physiological parameter after SPs with an SNR of 10-15 dBA occurring in quiet $(n=31)$ and active $(n=49)$ sleep depending on the time periods ( $\mathrm{a}^{*}$ and $\mathrm{b}^{*}$ ) after SPs

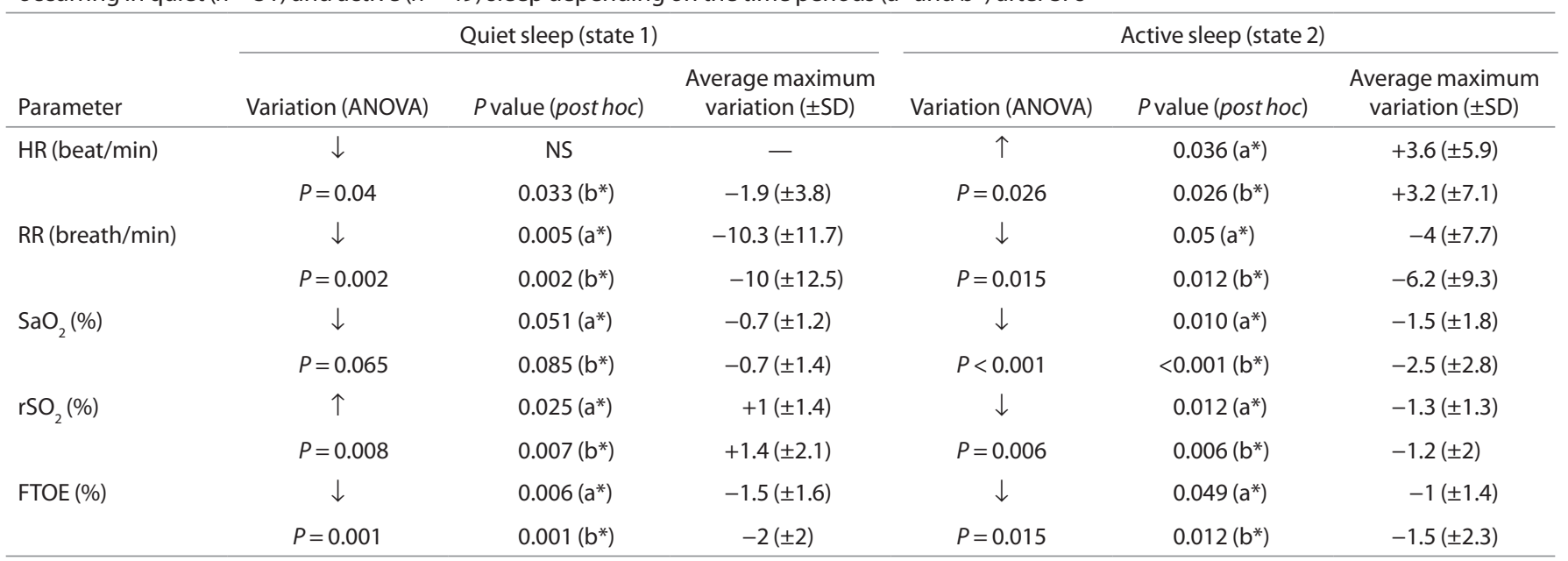

FTOE, fractional cerebral tissue oxygen extraction; $\mathrm{HR}$, heart rate; $\mathrm{NS}$, not significant; $\mathrm{RR}$, respiratory rate; $\mathrm{rSO}_{2^{\prime}}$, regional cerebral oxygen saturation; $\mathrm{SaO}{ }_{2}$, oxygen saturation; $\mathrm{SNR}_{\text {, }}$ signal-to-noise ratio.

$\downarrow, \uparrow$, Arrows indicate the direction of the observed variations, associated $P$ values indicate statistical significance (ANOVA for repeated-measure tests).

$a^{*}$ and $b^{*}$ indicate the periods ( $a^{*}: 0-20 \mathrm{~s}, b^{*}: 20-40 \mathrm{~s}$, post SP) in which a significant variation of the parameter was found in post hoc analysis (Newman-Keuls test) as compared with baseline.

during the $40 \mathrm{~s}$ after the occurrences of SPs in quiet and active sleep for each parameter are presented in Figure 3.

Following an SP, there were significant variations in mean HR, regardless of sleep state and range. Specifically, we observed increased HR in range 5-10 dBA and state 2 and a decrease in state 1 and range 10-15 dBA. We also observed significant decreases in mean RR in state 1 for both SNR ranges. During active sleep, RR decreased for both SNR ranges. We also observed a significant increase, but of smaller amplitude, in range 5-10 dBA.

The mean $\mathrm{SaO}_{2}$ levels varied differently depending on the SNR range. No significant variations were observed in quiet 
sleep except that $\mathrm{SaO}_{2}$ tended to decrease in range 10-15 dBA. During active sleep, however, there were significant oxygen desaturations in both SNR ranges. We observed significant increases in mean $\mathrm{rSO}_{2}$ during quiet sleep for both $\mathrm{SNR}$

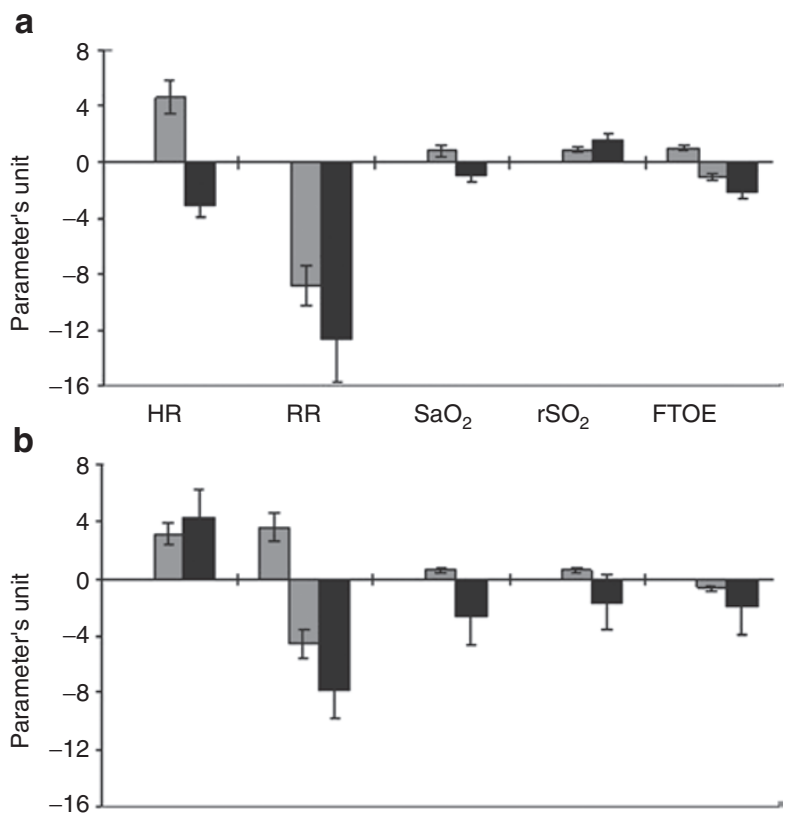

Figure 3. Means of the maximum significant changes of the physiological parameters as compared with baseline during the 40 s following SPs occurring in quiet (a) and active (b) sleep and according to SNR ranges (gray bar, 5-10 dBA; black bar, 10-15 dBA). Errors bars indicate standard errors. Parameter's units are as follow: beats/min for heart rate (HR); breaths/min for respiratory rate (RR); percentage (\%) for blood oxygen saturation $\left(\mathrm{SaO}_{2}\right)$, regional cerebral oxygen saturation $\left(\mathrm{rSO}_{2}\right)$, and fractional cerebral tissue oxygen extraction (FTOE). SNR, signal-to-noise ratio; SP, sound peak. ranges and for active sleep in range 5-10 dBA. By contrast, we observed a significant decrease in mean $\mathrm{rSO}_{2}$ for active sleep in range 10-15 dBA. Fractional cerebral tissue oxygen extraction (FTOE) decreased regardless of sleep state and SNR range, except for state 1 and range 5-10 dBA, where bidirectional reactivity was observed.

Main determinants of reactivity. To explore whether physiological reactivity was linked to the infant's gender, maturity, and postnatal experience (i.e., duration of noise exposure), we compared the response profiles of female and male infants, infants of postmenstrual age (PMA) $\geq 32(n=11)$ and $<32 \mathrm{wk}$, and infants of postnatal age $>15(n=15)$ and $\leq 15 \mathrm{~d}$ using a multilevel regression analysis model (Table 3 ). We observed only a few differences depending on these covariates. However, male infants (post hoc: $p<0.05, \mathrm{a}^{*}$ and $\mathrm{b}^{\star}$ ) and infants at younger PMA (post hoc: $p<0.02$, $\mathrm{a}^{\star}$ and $\mathrm{b}^{*}$ ) showed a greater increase in HR for SPs in range 5-10 dBA in active sleep. We also observed that younger infants showed greater decreases in $\mathrm{SaO}_{2}$ for SPs in range 5-10 dBA in quiet sleep (post hoc, $p=0.06 \mathrm{a}^{\star}$, and $p$ $\left.<0.05, b^{\star}\right)$. Conversely, more mature infants showed a greater decrease in $\mathrm{SaO}_{2}$ in active sleep for SPs in range 5-10 dBA (post hoc: $p=0.05, \mathrm{~b}^{\star}$ ) and for SPs in range 10-15 dBA (post hoc, $p$ $<0.03, \mathrm{a}^{*}$ and $\left.\mathrm{b}^{\star}\right)$. Finally, we found that increases in cerebral oxygen saturation (post hoc, $p<0.03, \mathrm{~b}^{*}$ ) and decrease in FTOE (post hoc, $p<0.02$, $\mathrm{a}^{\star}$ and $\mathrm{b}^{*}$ ) were greater for infants of younger postnatal age for SPs in range 10-15 $\mathrm{dBA}$ and in quiet sleep.

\section{DISCUSSION}

We evaluated the differential auditory sensitivities of VPIs to SPL increments by systematically and carefully examining their physiologic reactions to exogenous acoustic changes occurring

Table 3. Main determinants of the infants' reactivity depending on the sleep states and the SNR ranges

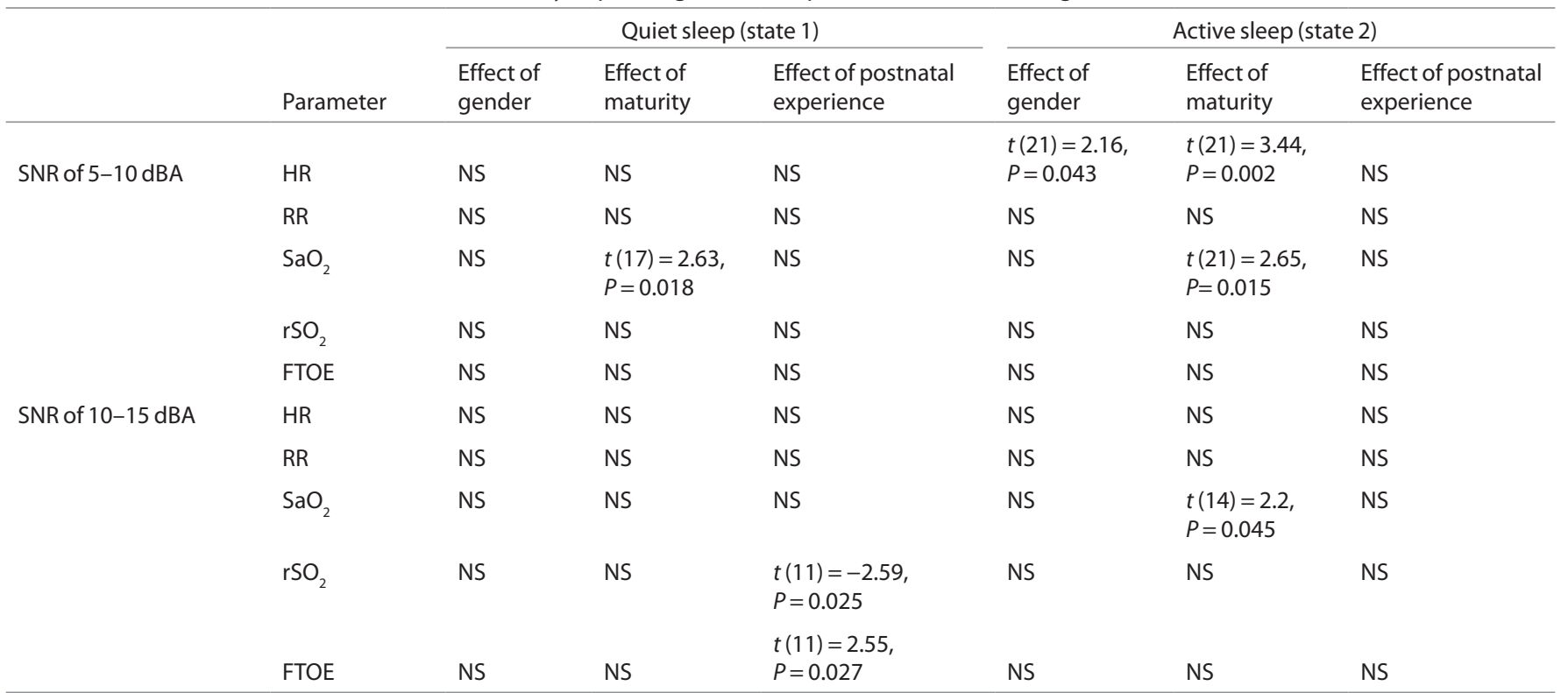

$P$ values indicate the result of a multilevel regression model analysis where physiological data are expressed as a function of gender, maturity, and postnatal experience. FTOE, fractional cerebral tissue oxygen extraction; $\mathrm{HR}$, heart rate; $\mathrm{NS}$, not significant; $\mathrm{RR}$, respiratory rate; $\mathrm{rSO}_{2}$, regional cerebral oxygen saturation; SaO $2^{\prime}$ oxygen saturation; $\mathrm{SNR}_{\text {, }}$ signal-to-noise ratio. 


\section{Articles | Kuhnetal.}

in their environment in the incubator. Our recordings are consistent with results of VPIs exposed to unpredictable, highfrequency (15), and intense noise in NICUs (16-19), with SPLs commonly exceeding the limits of $45 \mathrm{dBA}$ Leq recommended by the American Academy of Pediatrics (AAP) (5). Indeed, SPLs recorded weekly in incubators were recently found to exceed criteria more than $94 \%$ of the time (16). Our study provides additional details of the "naturalistic" acoustic environment of VPIs: they are exposed hourly to frequent variations in noise levels.

This study is an attempt to address some unresolved issues in the evaluation of the responses of premature newborns to the NICU naturalistic sound environment. Studies in this field are difficult to conduct because of practical limitations and technical and methodological challenges (12). One difficulty is that VPIs in incubators are continuously exposed to sensory inputs from multiple sources and of varying intensities and durations. In addition, changes in their respiratory support can influence their physiological parameters, and their behavioral state can alter their reactivity. We sought to minimize these variations as much as possible, both in study design (e.g., exclusion of periods during which VPIs could be exposed to tactile, vestibular, thermal, nociceptive, or visual stimuli) and in our analyses based on minimum and maximum values used to account for the variability in sound stimuli and individual responses. Although we enrolled only a relatively limited number of subjects, we analyzed a large number of SPs and systematically determined the newborns' initial arousal state before SPs occurred. To our knowledge, this is the first study to closely examine newborns' reactivity to naturally occurring sounds depending on their arousal state. We finally selected the SPs occurring during sleep because, in this state, infants' physiological parameters are less susceptible to variations caused by their motor activity, and SPs are not produced by the infant itself.

Our results, evaluating almost 600 SPL Leq increments between 5 and $15 \mathrm{dBA}$ above background noise during sleep of 26 VPIs, indicate that VPIs hear these stimuli with a minimal SNR threshold of 5-10 dBA. From at least 28 wk PMA, they react physiologically to auditory signals emerging from naturalistic background noise with median baseline SPL values varying, on average, between 50 and $59 \mathrm{dBA}$ Leq for newborns breathing room air or mechanically ventilated and newborns on nasal continuous positive airway pressure, respectively. An evaluation of developmental changes in masked thresholds in children, with the youngest being 6 mo old, showed that thresholds declined greatly as a function of age (20). Our findings are consistent with the known anatomical and functional development of the auditory system because previous studies using otoacoustic emission and brain stem response techniques have shown that the auditory system is functionally mature in PMAs as young as $30(21)$ and $28 \mathrm{wk}(22,23)$, respectively. The auditory system has been reported to be functional even by $25 \mathrm{wk}$ GA (24). This perceptual ability contributes to a minimal consciousness of the VPIs' environment (25).

How do VPIs react to these stimuli? The nature of the primary physiological responses (increased HR and decreased RR and oxygen saturation) and their timing (rapid onset during the $20 \mathrm{~s}$ after SP) suggest (i) a reflexive reaction, mediated by the brain stem, as shown for fetal cardiac reactivity to sounds and (ii) a stress (defensive) response, similar to those recorded for higher SPL stimuli $(8,9,13,26)$ and further emphasized by the emotional sweating that reflects sympathetic nervous system responses to a stressor after SPs in the same SPLs (14). To measure the impact of these stimuli on cerebral oxygenation, we used an near-infrared spectroscopy (NIRS) technique that can evaluate regional cortical activation (reflected by an increase in oxygen consumption accompanying neuronal activation) after sensory stimuli and has been used after auditory stimuli $(27,28)$. We noted significant but limited variations in these parameters. An increment in FTOE, a parameter reflecting variations in cerebral oxygenation independent of systemic oxygenation (29), corresponds to increased $\mathrm{O}_{2}$ consumption resulting from cerebral activation (30). Conversely, a decrease in FTOE indicates a decrease in oxygen consumption. Thus, the observed increases in both $\mathrm{rSO}_{2}$ and FTOE in state 1 for SPs in the 5- to 10-dBA range may suggest cerebral activation in the frontal cortex (28). The decreases in $\mathrm{rSO}_{2}$ and FTOE observed for higher SPLs during active sleep, although moderate, may suggest alterations in cerebral blood flow autoregulation caused by a stress reaction exceeding the homeostatic regulatory mechanisms.

Sleep/arousal states are important determinants of VPIs' reactivity. As already observed for auditory (31) and other sensory modalities (32), VPIs are more sensitive to sensory inputs during active sleep. Differences have been found to depend on the level of maturity and postnatal age, confirming that physiological responses to sound undergo age-dependent maturational changes, that postnatal experience may alter these physiological responses, and that, in contrast to full-term newborns, VPIs have an altered capacity to habituate to repeated sounds. More comprehensive studies are warranted. The impact of noise frequencies, known to be high in the NICU, and the nature of the sound source (i.e., human voices or artificial noise) on newborn responses and on the emotional valence that they attribute to these auditory stimuli must be examined. These questions should be addressed in future studies, which would benefit from an experimental design.

The exposure of vulnerable newborns to repeated, atypical, and improper noise, occurring during critical periods of brain development, can disrupt homeostatic regulatory mechanisms and can be harmful to VPIs. Longer-term consequences of exposure to deleterious auditory stimuli are more difficult to evaluate. Studies have failed to find a definite causative relationship between postnatal exposure to high noise and hearing loss. However, animal studies have shown that early auditory experience shapes sensory perception in mammals (33), can modify brain auditory mapping, and may retard auditory development (34).

\section{Conclusion}

In conclusion, our results provide further evidence of the auditory sensitivity of VPIs to their NICU environment. Their differential auditory sensitivities to SPL increments provide further evidence for the reinforcement of permissible noise 
criteria in the NICU $(7,35,36)$ and support recent modifications to these criteria (37). These findings also suggest not only that these recommendations should be expressed in terms of hourly Leq or maximum equivalent noise level over $1 \mathrm{~s}\left(\mathrm{~L}_{\max }\right)$ but also that efficient strategies should be developed and implemented to continuously lessen SPLs and/or attenuate their variations near VPIs or to protect infants from deleterious exposure to noise (38).

\section{METHODS}

\section{Participants and Environment}

A total of 26 VPIs were included from April 2008 to July 2009 in the NICU and the intermediate care unit of Strasbourg University Hospital, France. The study protocol was approved by our institutional review board, the Ethics Committee of Nancy, France (Comité de Protection des Personnes EST-III) on 1 April 2008. The parents of each infant provided written informed consent. Infants with major congenital anomalies or brain injuries and VPIs who had been administered analgesics/sedatives during the previous $48 \mathrm{~h}$ were excluded. The main characteristics of the study population are presented in Table 4. All infants were cared for in Draeger SC incubators (Draeger Medical, Lübeck, Germany). During the 10-h study period, all infants were lying in the prone position, on their backs, or on their sides. The study periods were similar for each newborn during the day. Basic developmental care measures were used, including incubator coverings, nesting, and general measures for sound abatement (lowering of alarm levels, minimizing talking by staff). However, there was no specific program for noise reduction. For study purposes, all staff members were encouraged to behave normally. Architecturally, the NICU comprised three four-bed rooms, one room with two beds, and one single-bed room. All rooms in the intermediate-care unit contained three beds.

\section{Procedures}

Environmental measurements. SPLs and environmental sounds were concomitantly recorded with a noise dosimeter (MS 6701-PRO Digital sound-level meter, Mastech, Pittsburgh, PA; measurement range $30-130 \mathrm{~dB}$, frequency range $30 \mathrm{~Hz}$ to $8 \mathrm{kHz}$ ) in A-weighted scale-slow response and with a microphone. The probes were placed at the same level, but at a $10-\mathrm{cm}$ distance, from each newborn's ear. According to standard recommendations, the probes were never in contact with the mattress. The probes were placed in incubator air,

Table 4. Characteristics of the study population

\begin{tabular}{|c|c|}
\hline Median [range] GA (wk) & $28[26-31]$ \\
\hline Mean ( \pm SD) birth weight (g) & $1109( \pm 250)$ \\
\hline Median postnatal age $(\mathrm{d})$ & $17[4-50]$ \\
\hline Median postmenstrual age (wk) & $31[28-34]$ \\
\hline Gender (girls-boys) ( $n$ ) & $10-16$ \\
\hline Small for GA/adapted for GA ( $n)$ & $8-18$ \\
\hline \multicolumn{2}{|c|}{ Respiratory support at time of study ( $n$ ) } \\
\hline Room air & 9 \\
\hline nCPAPa & 9 \\
\hline Mechanical ventilation ${ }^{\mathrm{b}}$ & 8 \\
\hline \multicolumn{2}{|c|}{ Median duration of respiratory support (d) } \\
\hline Oxygen supplementation & $16[0-36]$ \\
\hline nCPAPa & $6[0-30]$ \\
\hline Mechanical ventilation & $3[0-29]$ \\
\hline
\end{tabular}

$5 \mathrm{~cm}$ above the blanket. Luminance was simultaneously measured with a lux-meter (digital light meter RS180-7133, RS Components, Northants, UK) placed at the level of each newborn's eyes. SPLs and luminance were measured at frequencies of 25 and $0.5 \mathrm{~Hz}$, respectively. Leq was calculated directly by the sound-level meter and recorded at 1-s intervals. Light measurements were recorded in lux $\left(\mathrm{lm} / \mathrm{m}^{2}\right)$ at 2 -s intervals.

Physiological and cerebral data collection. HR, RR, and oxygen saturation $\left(\mathrm{SaO}_{2}\right)$ were continuously recorded by each infant's monitor using specific software (Dataplore CMS, ixellence $\mathrm{GmbH}$ Wildau, Germany). Cerebral oxygenation was assessed using a near infrared spectroscopy technique (INVOS 4100, Somanetics-Covidien, Paris, France), which measured regional cerebral oxygen saturation $\left(\mathrm{rSO}_{2}\right)$ calculated from the differential signal obtained over $5 \mathrm{~s}$ from a neonatal transducer fixed to each infant's frontal skull in the midline position (39). $\mathrm{rSO}_{2}$ was expressed as the venous-weighed percentage of oxygenated hemoglobin and FTOE was calculated using the formula $\left(\mathrm{FTOE}=\mathrm{SaO}_{2}-\left[\mathrm{rSO}_{2} / \mathrm{SaO}_{2}\right]\right)$.

Behavioral data collection. Each infant's face and body were video recorded with two video cameras (Sony color digital camera CCDB82A/S) placed outside the incubator and at the head and foot of the isolette, respectively, allowing subsequent analysis of body movements and facial expressions. Arousal states were assessed using Prechtl's observational rating system (40) by two specifically trained observers, who were unaware of the study's purpose: (i) eyes closed, regular respiration, no movements; (ii) eyes closed, irregular respiration, gross movements; (iii) eyes open, no gross movements; (iv) eyes open, continual gross movements, no crying; (v) eyes open or closed, fussing, or crying. Like Trapanotto et al. (11), we also included an intermediate state (vi) in which the eye positions, respiratory patterns, and movements did not match with any other state. The raters were allowed to stop and restart the video to establish a score. In case of conflicting evaluations, they subsequently performed a concomitant reevaluation to reach final agreement.

Data analyses. All data collected over a 10-h recording session were computerized and analyzed on the same time scale. To analyze auditory reactivity only, we excluded periods of direct skin contact with the newborn (routine care), periods in which light exposure varied $>10$ lux, and 1-min periods following any change in respiratory support. To better describe the acoustic environment of our study population, ambient noise levels were quantified by their 10th (L10), 50th (L50), and 90th (L90) percentiles in dBA Leq. According to the standard acoustic terminology, L90s and L10s are the lowest and highest deciles of noise levels, respectively. Because the decibel scale is logarithmic, $\mathrm{dBA}$ measurements were converted to sound pressure in Pascals, a linear scale, before averaging and the results were transformed again to dBA for presentation (16). To analyze acoustic changes, we identified SPs, which differ from the peaks (LpK) typically used in the acoustic literature. SPs were defined as a 1-s SPL (Leq) exceeding the previous 1-s interval by $5 \mathrm{dBA}$ Leq or more. Thus, the reference for SP measurements was the background SPL measured in dBA for each SP. They were identified using extracting data software (LabChart 7.1, Adinstrument, London, UK) and scanning the data every $2 \mathrm{~s}$. They were classified in different ranges based on 5-dBA Leq increments and reported as SNR on a dBA scale. They were also classified based on the newborn's sleep/arousal state at the time of SP occurrence. For each continuous variable, its mean value over $5 \mathrm{~s}$ was calculated for periods starting $5 \mathrm{~s}$ before each SP and for the following $40 \mathrm{~s}$.

\section{Statistical Analyses}

Each physiological parameter was compared using analysis of variance for repeated measures with "periods," sleep states, and SPs ranges as within factors and gender, GA, and postnatal age as between factors. Baseline values were first compared with the mean values obtained over 5 -s periods during the $40 \mathrm{~s}$ following SPs. To account for the effect of the great variations in the nature of SPs (differences in origins, durations, and frequencies of the stimuli) on the individual profile of response, baseline values were also compared with the minimum or maximum values obtained during the $20 \mathrm{~s}$ after each SP (period $a^{\star}$ ) 
and during the following $20 \mathrm{~s}$, from 21 to $40 \mathrm{~s}$ (period $b^{*}$ ). Post hoc analyses were performed when appropriate (Newman-Keuls test). Differences with $P \leq 0.05$ were considered statistically significant.

\section{ACKNOWLEDGMENTS}

We thank Agathe Rousselot, Laure Gorecky, and Manuela Cantarini for their invaluable help in collecting and reviewing the data and for behavioral analyses. We also thank the team of nurses for their assistance in acquiring data during the study period. We are especially grateful to the parents and their infants who participated in this research program.

\section{STATEMENT OF FINANCIAL SUPPORT}

No financial assistance was received in support of the study.

\section{REFERENCES}

1. Graven SN, Bowen FW Jr, Brooten D, et al. The high-risk infant environment. Part 1 . The role of the neonatal intensive care unit in the outcome of high-risk infants. J Perinatol 1992;12:164-72.

2. Anand KJ, Scalzo FM. Can adverse neonatal experiences alter brain development and subsequent behavior? Biol Neonate 2000;77:69-82.

3. Philbin MK, Lickliter R, Graven SN. Sensory experience and the developing organism: a history of ideas and view to the future. J Perinatol 2000;20(8 Pt 2):S2-5.

4. Als H, Duffy FH, McAnulty GB, et al. Early experience alters brain function and structure. Pediatrics 2004;113:846-57.

5. American Academy of Pediatrics. Committee on Environmental Health 1997 Noise: a hazard for the fetus and newborn. Pediatrics 1997;100:724-7.

6. Philbin MK, Robertson A, Hall JW 3rd. Recommended permissible noise criteria for occupied, newly constructed or renovated hospital nurseries. The Sound Study Group of the National Resource Center. J Perinatol 1999;19(8 Pt 1):559-63.

7. White RD. Recommended standards for the newborn ICU. J Perinatol 2007;27 Suppl 2:S4-S19.

8. Wharrad HJ, Davis AC. Behavioural and autonomic responses to sound in pre-term and full-term babies. Br J Audiol 1997;31:315-29.

9. Vranekovic G, Hock E, Isaac P, Cordero L. Heart rate variability and cardiac response to an auditory stimulus. Biol Neonate 1974;24:66-73.

10. Long JG, Lucey JF, Philip AG. Noise and hypoxemia in the intensive care nursery. Pediatrics 1980;65:143-5.

11. Trapanotto M, Benini F, Farina M, Gobber D, Magnavita V, Zacchello F. Behavioural and physiological reactivity to noise in the newborn. J Paediatr Child Health 2004;40:275-81.

12. Wachman EM, Lahav A. The effects of noise on preterm infants in the NICU. Arch Dis Child Fetal Neonatal Ed 2011;96:F305-9.

13. Williams AL, Sanderson M, Lai D, Selwyn BJ, Lasky RE. Intensive care noise and mean arterial blood pressure in extremely low-birth-weight neonates. Am J Perinatol 2009;26:323-9.

14. Salavitabar A, Haidet KK, Adkins CS, Susman EJ, Palmer C, Storm H. Preterm infants' sympathetic arousal and associated behavioral responses to sound stimuli in the neonatal intensive care unit. Adv Neonatal Care 2010;10:158-66.

15. Livera MD, Priya B, Ramesh A, et al.; Swarnarekha. Spectral analysis of noise in the neonatal intensive care unit. Indian J Pediatr 2008;75:217-22.

16. Lasky RE, Williams AL. Noise and light exposures for extremely low birth weight newborns during their stay in the neonatal intensive care unit. Pediatrics 2009;123:540-6.

17. Benini F, Magnavita V, Lago P, Arslan E, Pisan P. Evaluation of noise in the neonatal intensive care unit. Am J Perinatol 1996;13:37-41.

18. Philbin MK. The influence of auditory experience on the behavior of preterm newborns. J Perinatol 2000;20(8 Pt 2):S77-87.
19. Williams AL, van Drongelen W, Lasky RE. Noise in contemporary neonatal intensive care. J Acoust Soc Am 2007;121(5 Pt1):2681-90.

20. Schneider BA, Trehub SE, Morrongiello BA, Thorpe LA. Developmental changes in masked thresholds. J Acoust Soc Am 1989;86:1733-42.

21. Morlet T, Collet L, Duclaux R, et al. Spontaneous and evoked otoacoustic emissions in pre-term and full-term neonates: is there a clinical application? Int J Pediatr Otorhinolaryngol 1995;33:207-11.

22. Lary S, Briassoulis G, de Vries L, Dubowitz LM, Dubowitz V. Hearing threshold in preterm and term infants by auditory brainstem response. J Pediatr 1985;107:593-9.

23. Ponton CW, Moore JK, Eggermont JJ. Auditory brain stem response generation by parallel pathways: differential maturation of axonal conduction time and synaptic transmission. Ear Hear 1996;17:402-10.

24. Hall JW 3rd. Development of the ear and hearing. J Perinatol 2000;20(8 Pt 2):S12-20.

25. Lagercrantz H, Changeux JP. The emergence of human consciousness: from fetal to neonatal life. Pediatr Res 2009;65:255-60.

26. Lagercrantz H, Edwards $D$, Henderson-Smart D, Hertzberg $T$, Jeffery H. Autonomic reflexes in preterm infants. Acta Paediatr Scand 1990;79:721-8.

27. Sakatani K, Chen S, Lichty W, Zuo H, Wang YP. Cerebral blood oxygenation changes induced by auditory stimulation in newborn infants measured by near infrared spectroscopy. Early Hum Dev 1999;55:229-36.

28. Zaramella P, Freato F, Amigoni A, et al. Brain auditory activation measured by near-infrared spectroscopy (NIRS) in neonates. Pediatr Res 2001;49:213-9.

29. Naulaers G, Meyns B, Miserez M, et al. Use of tissue oxygenation index and fractional tissue oxygen extraction as non-invasive parameters for cerebral oxygenation. A validation study in piglets. Neonatology 2007;92:120-6.

30. Naulaers G, Morren G, Van Huffel S, Casaer P, Devlieger H. Cerebral tissue oxygenation index in very premature infants. Arch Dis Child Fetal Neonatal Ed 2002;87:F189-92.

31. Trinder J, Newman NM, Le Grande M, et al. Behavioural and EEG responses to auditory stimuli during sleep in newborn infants and in infants aged 3 months. Biol Psychol 1990;31:213-27.

32. Marlier L, Schaal B, Gaugler C, Messer J. Olfaction in premature human newborns: detection and discrimination abilities two months before gestational term. In: Marchlewska-Koj A, Lepri JJ, Müller-Schwarze D, eds. Chemical Signals in Vertebrates 9. New York: Kluwer Academic/Plenum, 2001:205-9.

33. Han YK, Köver H, Insanally MN, Semerdjian JH, Bao S. Early experience impairs perceptual discrimination. Nat Neurosci 2007;10:1191-7.

34. Chang EF, Merzenich MM. Environmental noise retards auditory cortical development. Science 2003;300:498-502.

35. Graven SN. Sound and the developing infant in the NICU: conclusions and recommendations for care. J Perinatol 2000;20(8 Pt 2):S88-93.

36. Liu WF, Laudert S, Perkins B, Macmillan-York E, Martin S, Graven S; NIC/Q 2005 Physical Environment Exploratory Group. The development of potentially better practices to support the neurodevelopment of infants in the NICU. J Perinatol 2007;27 Suppl 2:S48-74.

37. Philbin MK, Evans JB. 2006 Standards for the acoustic environment of the newborn ICU. J Perinatol 26:S27-30.

38. Abou Turk C, Williams AL, Lasky RE. A randomized clinical trial evaluating silicone earplugs for very low birth weight newborns in intensive care. J Perinatol 2009;29:358-63.

39. Toet MC, Lemmers PM, van Schelven LJ, van Bel F. Cerebral oxygenation and electrical activity after birth asphyxia: their relation to outcome. Pediatrics 2006;117:333-9.

40. Prechtl HF. The behavioural states of the newborn infant (a review). Brain Res 1974;76:185-212. 